\title{
Influence of Field of View in Visual Prostheses Design: Analysis with a VR System
}

\author{
Melani Sánchez-García, Rubén Martínez-Cantín, Jesús Bermúdez-Cameo, Jose J. \\ Guerrero
}

\author{
Grupo de robótica, percepción y tiempo real (RoPeRT) \\ Instituto de Investigación en Ingeniería de Aragón (I3A) \\ Universidad de Zaragoza, Mariano Esquillor s/n, 50018, Zaragoza, Spain. \\ Tel.+34-976762707, e-mail: mesangar@unizar.es
}

\begin{abstract}
In this work, we evaluate the influence of field of view with respect to spatial resolution in visual prostheses. Our system uses a virtual-reality environment based on panoramic scenes and headmounted display which allows users to feel immersed in the scene. Results indicate that, for the design of retinal prostheses, it is better to concentrate the phosphenes in a small area, to maximize the angular resolution, even if that implies sacrificing field of view.
\end{abstract}

\section{Introduction}

Retinitis pigmentosa and age-related macular degeneration are two important causes of visual impairment and blindness [1]. Retinal prostheses are a promising technology to improve vision in patients with such advanced degenerative diseases [2]. These visual prostheses can partially restore vision, bypassing damaged photoreceptors and electrically stimulating the surviving retinal cells, such as the retinal ganglion cells. However, there are still physiological and technological limitations of the information received by implanted patients such as the resolution (up to 1500 phosphenes), dynamic range of the visual perception (8 gray levels) and the field of view (FOV) of approximately $18^{\circ} \times 11^{\circ}$ (see Figure 1). To improve the design of current visual prostheses in terms of FOV and resolution several devices for retinitis pigmentosa patients (tunnel vision) have been designed to expand the projected FOV on their retina, for example, minimizing the scene zooming out using a camera, using thermal imaging by changing the mapping (zoom out) between the sensor and the electrode array or by spreading the electrodes in a wider retinal surface [3]. Researchers have previously used simulated prosthetic vision (SPV) for analysis of the visual perception in terms of resolution or FOV with normal sight subjects [4]. Most of the experiments relied on some form of head-mounted device which allowed a virtual- reality experience during the experiment, but the immersiveness of experience was poor due to the technological limitations compared to modern commercial VR systems.

In this work, we evaluated the influence of FOV and resolution of the prostheses on the subject's performance in a recognition task, since it is of high priority for patients with visual diseases such as retinitis pigmentosa. Concretely, we analyzed an object search and recognition task performance in indoor scenes with different reduced FOVs and resolutions limited to hundreds of electrodes. For that, we present a new VR system for more realistic SPV environments using panoramic scenes. The VR system could be extended to more complex tasks because it supports realistic environments. This system acts as an electronic visual aid that attach to the user's head and presents information directly to the user's eyes. The panoramic scenes allow subjects felt immersed in the scene by perceiving the entire scene (360 degrees).

\section{Simulated prosthetic vision (SPV)}

Our SPV system includes hardware specifications, software components and phosphene generation. The representation with simulated phosphenes was displayed on the VR system worn by the participants a well as on the laptop screen for the experimenter to check the progress. During the experiment, participants were seated in a swivel chair allowing them to scan the entire scene with head rotation movements. Starting from a panoramic scene of $360^{\circ}$ of horizontal FOV and $180^{\circ}$ of vertical FOV, the system estimates the orientation of the user using the information collected from the IMU. The selected area is then projected on the two Oculus displays and finally represented with simulated phosphenes (with a Gaussian luminance profile and 8 intensity levels). 
Our software to simulate the prosthetic vision with the VR system is available at http://webdiis.unizar.es/ rmcantin/index.php/Resea rch/Vrfov.

For the formal experiment, participants were recruited to complete an object search and recognition task using the SPV system. The scenes were presented to the subjects using different stimuli conditions based on two resolutions (200 and 500 phosphenes) and three FOVs (20, 40 and 60 degrees), as can be seen in Figure 2.

\section{Conclusions}

We have analyzed the influence of field of view with respect to resolution in visual prostheses through a study with a novel simulated prosthetic vision setup: a virtual-reality system using panoramic scenes. Our results show that, for the same number of phosphenes, recognition accuracy and response time improved by reducing the field of view. In fact, angular resolution is major determinant for effective object recognition, being directly correlated to the accuracy and inversely correlated to the response time. However, it has also shown a diminishing return even for an angular resolution of less than 2.3 phosphenes per degree. Simulated prosthetic vision allows experiments with larger number of participants and simpler procedures than clinical studies with implanted patients. Our experimental setup relies on a consumer-level head-mounted display, public image databases and we have released the software needed to run the simulator, to facilitate replications and extensions. Our results seem to indicate that it is better to concentrate the phosphenes to maximize the angular resolution, sacrificing field of view.

\section{REFERENCES}

[1]. Hartong D T, Berson E L and Dryja T P 2006 The Lancet3681795-1809

[2]. Zhou D D, Dorn J D and Greenberg R J 2013 The Argus II retinal prosthesis system: An overview IEEE International Conference on Multimedia and Expo Workshops (ICMEW) pp 1-6

[3]. He Y, Huang N T, Caspi A, Roy A and Montezuma S R 2019 Translational vision science \& technology 829-29

[4]. Sanchez-Garcia M, Martinez-Cantin R and Guerrero J J 2020 PloS one $15 \mathrm{e} 0227677$

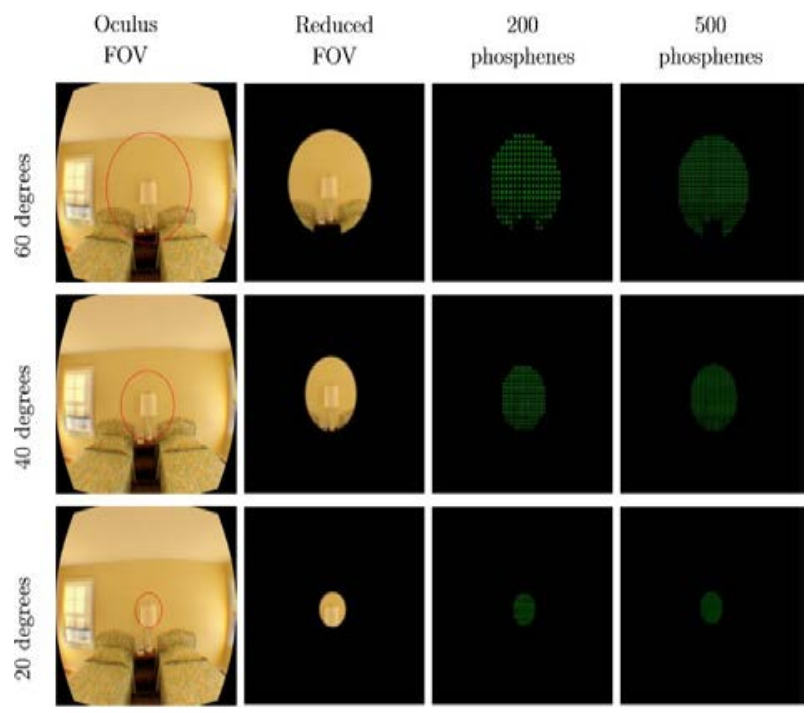

Figure 2: Stimuli conditions in the experiment. Rows: different FOVs used in the experiment (60, 40 and 20 degrees). Colums: different resolutions used in the experiment $(200$ and 500 phosphenes). Note that in the last row only part of the lamp is visible, the beds cannot be seen without moving the point of view.

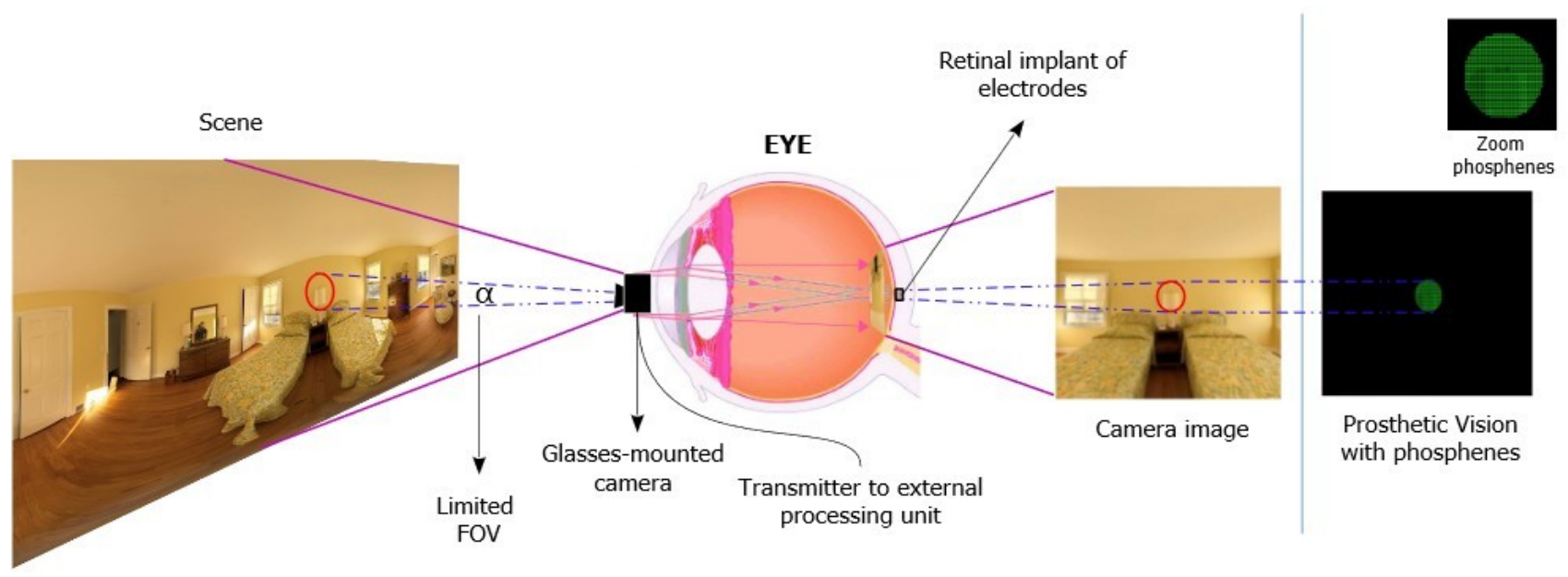

Figure 1: Overview of a retinal prosthesis. The external and internal components include a glasses-mounted camera, an external processing unit and an implanted electrode array. First, the external camera acquires an image. Then, the external processor converts the image to a suitable pattern of electrical stimulation of the retina through an electrode array. The result is a phosphene image with limited field of view (FOV).

Revista “Jornada de Jóvenes Investigadores del I3A”, vol. 8 (Actas de la IX Jornada de Jóvenes Investigadores del I3A -11 de diciembre de 2020). ISSN 2341-4790. 